\title{
Two MYB transcription factors (CsMYB2 and CsMYB26) are involved in flavonoid biosynthesis in tea plant [Camellia sinensis (L.) O. Kuntze]
}

Wen-Li Wang ${ }^{\dagger}$, Yong-Xin Wang ${ }^{\dagger}$, Hui Li, Zhi-Wei Liu, Xin Cui and Jing Zhuang ${ }^{*}$

\begin{abstract}
Background: Flavonoids are secondary metabolites that play important roles in the entire tea plant life cycle and have potential health-promoting properties. MYB transcription factors (TFs) are considered potentially important regulators of flavonoid biosynthesis in plants. However, the molecular mechanisms by which MYB TFs regulate the flavonoid pathway in tea plant remain unknown.

Results: In this study, two R2R3-MYB TFs (CSMYB2 and CsMYB26) involved in flavonoid biosynthesis in tea plant were investigated. The genes encoding CsMYB2 and CsMYB26 were cloned from the tea plant cultivar 'Longjing 43'. Phylogenetic analysis showed that CsMYB2 and CsMYB26 were grouped into the proanthocyanidin biosynthesisrelated MYB clade. Multiple sequence alignment revealed that conserved motif 1 in the two MYB factors was related to the bHLH TF. Subcellular localization assays suggested that CSMYB2 localized in the nucleus. Promoter analysis indicated that CSMYB2, CSMYB26 and the related structural genes contain MYB recognition elements. The expression levels of the CSMYB2 and CSMYB26 genes and the structural genes in the flavonoid biosynthesis pathway were determined in leaves from various sites in the two tea plant cultivars 'Longjing 43' and 'Baiye 1 hao'.

Conclusions: The expression levels of these genes were correlated with the accumulated flavonoid content. The results demonstrated that the expression level of CSF3'H may be regulated by CSMYB2 and that CSMYB26 expression is positively correlated with CSLAR expression. The relative transcriptional level of CSMYB26 may be the main reason for the different epigallocatechin contents between the tea plant cultivars 'Longjing 43' and 'Baiye 1 hao'. Our results will serve as a reference for the potential regulatory roles of CSMYB2 and CsMYB26 in flavonoid biosynthesis in tea plant and may also assist biologists in improving tea quality.
\end{abstract}

Keywords: Tea plant, Flavonoid biosynthesis, CsMYB2, CsMYB26, Subcellular localization, Transcriptional level

\section{Background}

Tea plant [Camellia sinensis (L.) O. Kuntze] is an important economic leaf crop worldwide, and tea is known as a healthy beverage [1]. In tea plants, catechins, anthocyanidins, and proanthocyanidins (PAs) are important secondary metabolites that are synthesized via the flavonoid pathway.

\footnotetext{
* Correspondence: zhuangjing@njau.edu.cn

The original version of this article was revised: the mismatch between figures and their legends has been corrected.

'Wen-Li Wang and Yong-Xin Wang contributed equally to this work.

Tea Science Research Institute, College of Horticulture, Nanjing Agricultural University, 1 Weigang, Nanjing 210095, Jiangsu, China
}

As one of the most researched secondary metabolism pathways in tea plant growth processes, the flavonoid pathway is divided into the "early" flavonoid pathway and the "late" flavonoid pathway [2]. In the "early" flavonoid pathway, the synthesis of different metabolites shares the same structural genes and biosynthetic precursors, namely, chalcone synthase $(C H S)$, chalcone isomerase $(C H I)$, flavanone 3-hydroxylase $(F 3 H)$, flavanone 3'-hydroxylase $\left(F 3^{\prime} H\right)$, and flavanone 3',5' -hydroxylase $\left(F 3^{\prime} 5^{\prime} H\right)$. Correspondingly, the biosynthesis of the different secondary metabolites catechin, anthocyanin, and PA is mainly controlled by the "late" flavonoid 
pathway. For example, the leucoanthocyanidin reductase $(L A R)$ gene is key for the production of catechin $(C)$; anthocyanidin synthase (ANS) and anthocyanidin reductase $(A N R)$ are important structural genes for the production of epicatechin (EC), gallocatechin (GC), epigallocatechin (EGC), epicatechin gallate, and epigallocatechin gallate. Different catechin monomers are substrates for UDP-glucose:flavonoid 3-O-glucosyltransferase (UFGT) in the production of anthocyanins and the hydroxylation and polymerization of flavan-3-ol to form PAs $[3,4]$.

In higher plants, flavonoid biosynthesis is not only regulated by structural genes but also involves a number of regulatory genes $[5,6]$. Studies have demonstrated that different transcription factor (TF) families, such as the MYB, bHLH, and WD40 families, physically interact to form the MBW complex and are responsible for regulating flavonoid biosynthesis [6, 7].

The MYB family is one of the largest TF families in plants. The R2R3-MYB-type family contains the largest number of members. In Arabidopsis thaliana, according to functional analysis, AtR2R3-MYB-type family members are divided into 25 subgroups $[8,9]$. Members of the MYB 5 subgroup mainly control PA metabolism in the flavonoid pathway; however, to date, only AtMYB123 has been found to belong in this subgroup in A. thaliana [10]. In the R2R3-MYB-type TF family, the conserved motif (DNEI $[\mathrm{A} / \mathrm{S} / \mathrm{G}] \mathrm{N}[\mathrm{D} / \mathrm{A} / \mathrm{N}] \mathrm{V}$ ) was proven to bind to a specific site in BHLH. Moreover, this motif has been found in the R3 structural domain $[9,11]$. The $\mathrm{N}$-terminal MYB domains are very conserved, in the $\mathrm{N}$-terminal but those in the $\mathrm{C}$-terminal vary. The C-terminal often contains transcriptional activation or repression domains as well as conserved serine and threonine residues, which may correspond to posttranslational modification sites [12].

The sequence features of R2R3-MYB TFs and the regulatory activities of these TFs in the flavonoid pathway have been investigated in various plants [13-17]. Nathalie et al. found that TT2 at least partially determined PA accumulation [10]. The overexpression of VvMYBPA1 in Arabidopsis induced the upregulation of flavonoid pathway genes and the accumulation of PAs [18]. Laurent et al. elucidated the ability of the $V v M Y B 5 a$ gene to be mainly expressed during the early stages of berry development in skin, flesh, and seeds. The overexpression of $V v M Y B 5 a$ in tobacco affected the expression profiles of structural genes controlling the synthesis of phenylpropanoids and the metabolism of anthocyanins [19]. There is a positive linear correlation between PPMYB10 and PpMYBPA1 and the metabolites of the flavonoid pathway. Ravaglia et al. concluded that anthocyanins are regulated by MYB10 and that PAs are regulated by MYBPA1 [20]. Xu et al. found that
DcMYB6 TFs are involved in regulating anthocyanin biosynthesis in purple carrot taproots [6]. An R2R3-MYB TF, OjMYB1, was reported to be involved in anthocyanin biosynthesis in Oenanthe javanica [5]. AgMYB2 is involved in the regulation of anthocyanin biosynthesis in purple celery [21]. MdMYBA induces anthocyanin accumulation in the reproductive tissues of transgenic tobacco [22]. CsMYB4a is potentially involved in the regulation of flavonoid gene expression [23]. CsAN1 was isolated from the 'Zijuan' cultivar and found to regulate anthocyanin accumulation [13].

R2R3 MYB TFs are essential for plants and are believed to play important roles in regulating the flavonoid pathway in various species [6, 13, 24-26]. However, the molecular mechanisms by which MYB TFs regulate the flavonoid pathway in tea plant remain unknown. In the present study, we investigated two MYB TFs (CsMYB2 and CsMYB26) involved in the flavonoid metabolism pathway in tea plant. In addition, we collected leaves from different sites on two tea plant cultivars, 'Longjing 43 ' and 'Baiye 1 hao'. The former cultivar is a stable early budding cultivar, while the latter is a typical low temperature-sensitive small leaf cultivar. Different metabolites (catechins, anthocyanins, and PAs) in the flavonoid pathway were detected. Our results will provide a reference on how MYB TFs regulate the flavonoid pathway in tea plant and may also assist biologists in improving tea plant quality.

\section{Results}

Cloning of CsMYB2 and CsMYB26 genes from tea plant The CsMYB2 and CsMYB26 genes were cloned from the tea plant cultivar 'Longjing 43'. The amplification products and open reading frames (ORFs) of the cloned sequences comprised 924 and $921 \mathrm{bp}$, respectively. The integral nucleotide sequences and deduced amino acid sequences are shown in Fig. 1.

\section{Phylogenetic tree analysis and conserved motif analysis of the CsMYB2 and CsMYB26 TFs}

To analyse the evolutionary relationships of CsMYB2 and CsMYB26 in tea plant with those in Arabidopsis, an unrooted phylogenetic tree was constructed. As shown in Fig. 2, CsMYB2 and CsMYB26 were classified into subgroup 5. CsMYB2 and CsMYB26 were closely related to AtMYB123, which is involved in the flavonoid pathway. The amino acid sequences of homologs involved in the flavonoid pathway from various species, including FaMYB9 and FaMYB11 from strawberry (Fragaria ananassa); MdMYB9 and MdMYB11 from apple (Malus domestica); FcMYB251 from Japanese beech (Fagus crenata); VvMYBPAR, VvMYBPA2, and VvMYBPA1 from grapevine ( $V$. vinifera); LjTT2a from lotus (Lotus japonicas); AtMYB123 from Arabidopsis; OsMYB3 from 


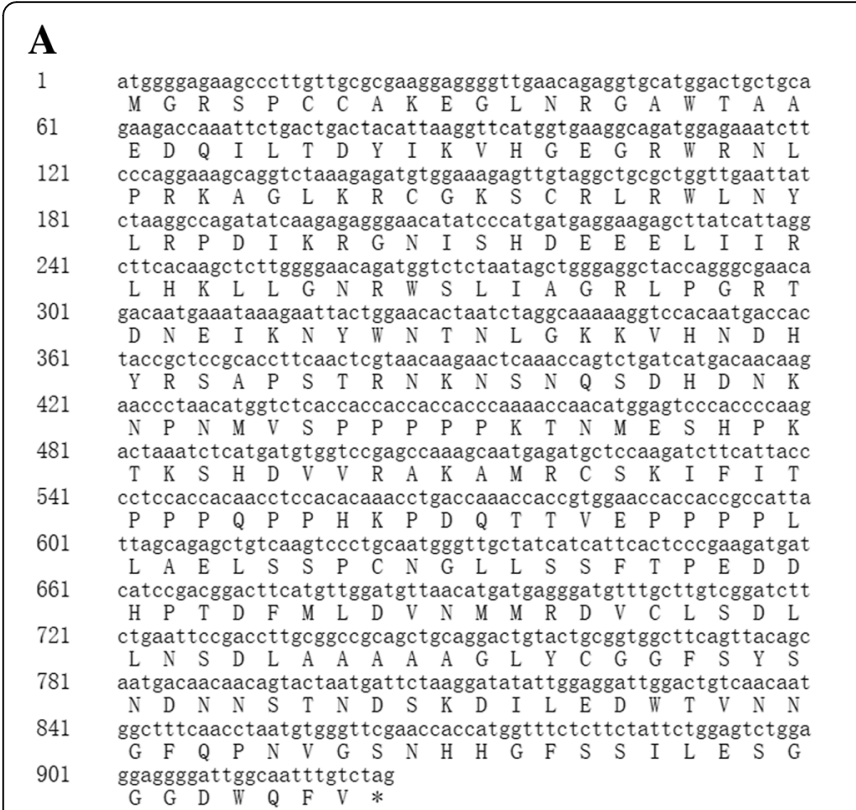

B

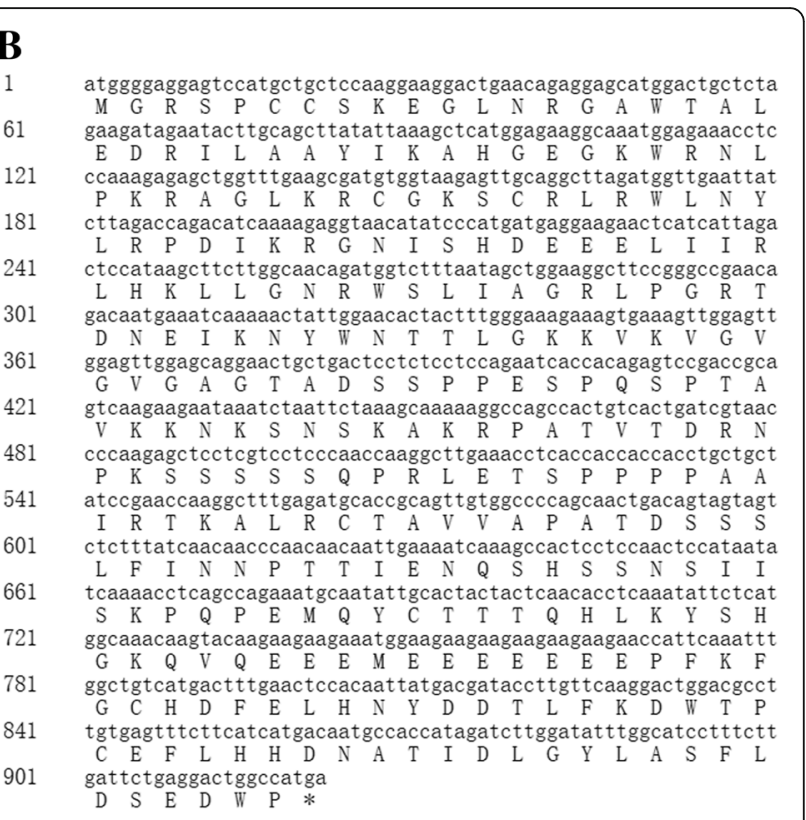

Fig. 1 Gene sequences of CSMYB2 and CSMYB26 with the deduced amino acid sequences. (a) CSMYB2 gene. (b) CsMYB26 gene

rice (Oryza sativa); and DkMYB2 and DkMYB4 from persimmon (Diospyros kaki), were selected to construct the phylogenetic tree. The phylogenetic analysis indicated that CsMYB2 and CsMYB26 have markedly orthologous relationships with VvMYBPAR and FcMYB251, respectively. CsMYB2 and CsMYB26 were grouped into the PA biosynthesis-related MYB clade (Fig. 3).

The TFs involved in the PA pathway, along with the CsMYB TFs were selected, and multiple sequence alignment was performed. Figure 4 shows that the R2 and R3 domains were conserved in these species and that different sequences of motif1 and motif 2 from the same subgroup were highly conserved. Moreover, motif1 is also known as the bHLH motif, which is required for the interaction with the bHLH protein.

\section{Subcellular localization analysis of CsMYB2}

The ORF of the CsMYB2 gene was inserted into the GFP reporter gene under the control of the CaMV $35 \mathrm{~S}$ promoter. The CsMYB2-GFP fusion gene and GFP recombinant constructs, as controls, were introduced into onion epidermal cells by particle bombardment. The results showed that the CsMYB2-GFP fusion protein was specifically localized in the nucleus (Fig. 5). The GFP signal from the empty vector showed ubiquitous distribution throughout the cell.

Interaction network of CsMYB2, CsMYB26 and the structural genes involved in the flavonoid pathway in tea plant

To better understand the interactions among CsMYB2, CsMYB26 and the structural genes involved in the flavonoid pathway in tea plant, an interaction network was built using STRING software on the basis of the orthologs in Arabidopsis (Fig. 6). MYB12 (CsMYB2) is a flavonol-specific activator of flavonoid biosynthesis that can activate the expression of $\mathrm{CHS}, \mathrm{CHI}, \mathrm{F} 3 \mathrm{H}$, and FLS1. MYB12 (CsMYB2) interacts with BAN (CsANR and CsLAR), UGT78D2 (CsUFGT), TT7 (CsF3'H), DFR (CsDFR), and LDOX (CsANS), while MYB2 (CsMYB26) only interacts with DFR (CsDFR).

\section{Analysis of the promoter regions of CsMYB2, CsMYB26 and the structural genes involved in the flavonoid pathway in tea plant}

The sequences of CsMYB2, CsMYB26 and the related structural genes (CsF3'H, CsDFR, CsANS, CsANR, CsLAR, and CsUFGT) 2000 bp upstream of the transcription start site were analysed to understand the regulatory mechanisms that control the expression of these genes. As shown in (Additional file 1), several regulatory elements, which are related to important physiological processes, such as the light response, hormonal/environment responses, and developmental regulation, were found in the promoter regions. Several light-responsive elements were widely present in the promoter regions, including Box4, ACE, G-box, GT1 motif, and TCT motif elements. This finding suggested that the expression of these genes might be related to photosynthesis and carbohydrate metabolism. Moreover, cis-regulatory elements were present in the promoter regions of CsMYB2, CsMYB26, CsF3'H, CsDFR, CsANS, CsANR, CsLAR, and CsUFGT. These elements are related to the signaling pathways of abscisic acid (ABA), ethylene (ERE), methyl jasmonic acid (MeJA), jasmonic 


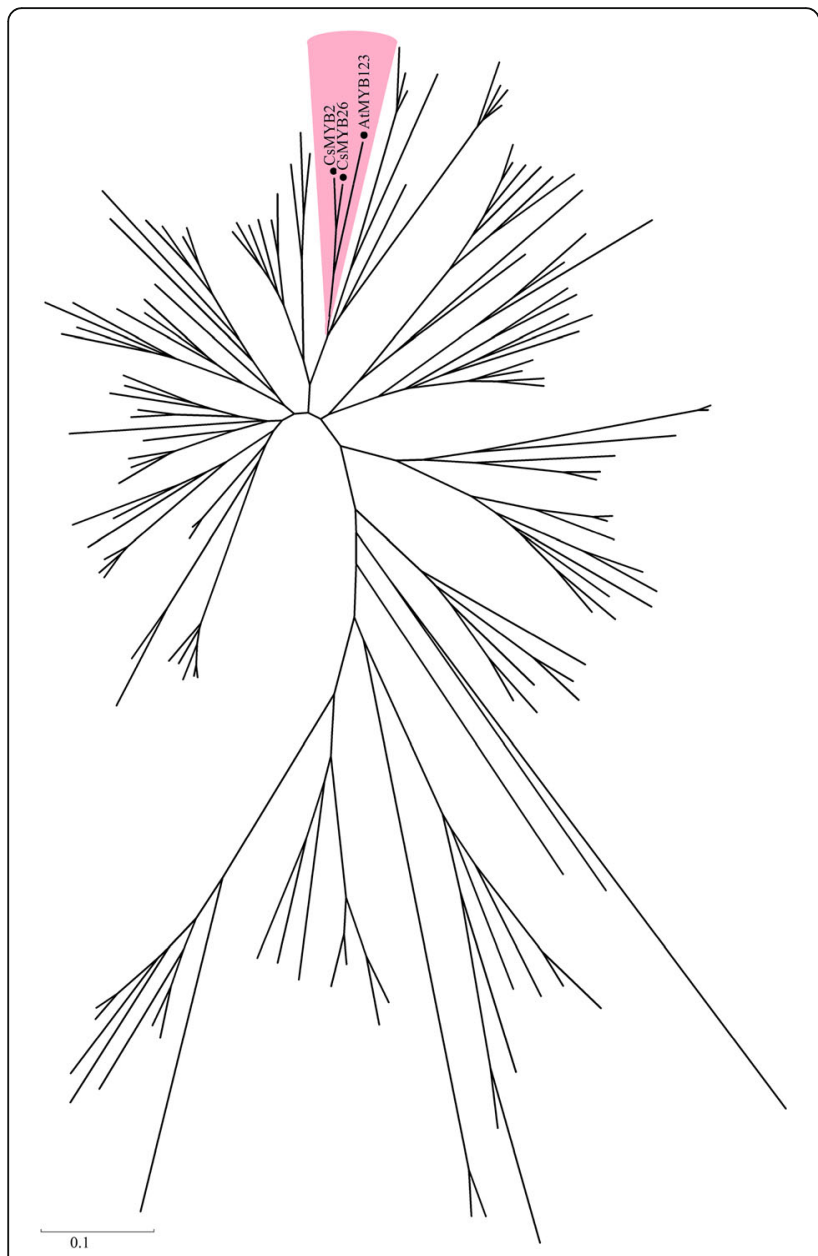

Fig. 2 Unrooted phylogenetic tree of CSMYB2 and CsMYB26 with R2R3-MYB-type A. thaliana TFs. A phylogenetic tree was built using the neighbor-joining method with MEGA 5 software. The putative functions of all R2R3-MYBs are listed on the right

acid (SA), and auxin. Among these elements, ABA, SA, and MeJA are important signaling molecules in plant responses to stress, indicating that most of these genes are involved in responses to biotic and abiotic stresses. The promoters of these genes contain many MBS, ARE, W box, STRE, and TC-rich repeat elements, indicating that these genes are subject to stress regulation. Additionally, the promoters of these genes contain many MYB recognition site elements. Among these elements, MYB recognition sites were found in all genes examined. The results suggested that the expression of structural genes might be regulated by $C s M Y B$ genes and that $C s M Y B 2$ and CsMYB26 might also be regulated by other CsMYB genes.

\section{Expression profiles of CsMYB2, CsMYB26 and the} structural genes under $A B A$ and shading treatments The expression levels of CsMYB2 and CsMYB26 along with those of the structural genes involved in the flavonoid pathway (CsF3'H, CsDFR, CsANS, CsANR, CsLAR, and CsUFGT) were determined under $\mathrm{ABA}$ and shading treatments (Fig. 7).

\section{ABA treatment}

As shown in Fig. 7a, almost all tested genes were gradually downregulated. However, CsLAR was first upregulated at 2 and $4 \mathrm{~h}$ and was then gradually downregulated.

\section{Shading treatment}

The expression levels of most tested genes were increased with increasing time in both sunlight and shade conditions (Fig. 7b). The expression levels of CsMYB2, CsMYB26, CsDFR, CsANS, CsANR, and CsUFGT were lower under shading treatment than under sunlight treatment. Conversely, CsF3'H and CsLAR exhibited higher expression levels under shading treatment than under sunlight treatment.

\section{Relationships among the expression profiles of CsMYB2, CsMYB26 and the structural genes involved in the flavonoid pathway}

As shown in Fig. 8, the expression levels of the CsMYB2 gene for leaves from different sites on the plants were not significantly different between 'Longjing 43' and 'Baiye 1 hao'. The successive decreasing order of the expression levels was tender leaves $>$ older leaves $>$ mature leaves. In addition, mature leaves and older leaves showed no significant difference in gene expression profiles. The CsMYB26 gene showed the lowest expression levels in old leaves and the highest expression levels in tender leaves.

The expression levels of genes encoding the enzymes in the flavonoid pathway (CsF3'H, CsDFR, CsANS, CsANR, CsLAR, and CsUFGT) were determined in the leaves at different sites (Fig. 9). The expression profile of $C s F 3^{\prime} H$ was in accordance with that of CsMYB2. In addition, the gene expression levels in 'Longjing 43' were higher than those in 'Baiye 1 hao'. In 'Baiye 1 hao', the expression profiles of CsDFR, CsANS, and CsLAR were in accordance with the CsMYB26 expression levels. The highest expression levels were found in tender leaves, and the lowest expression levels were found in older leaves. However, this consistent pattern was not found in 'Longjing 43'. In 'Longjing 43', CsDFR and CsANR had the highest expression profiles in mature leaves and tender leaves, with lower expression levels in tender leaves and mature leaves. The difference between the two tea plant cultivars suggested that the gene expression patterns may be due to cultivar specificity.

\section{Catechin (GC, EGC, C, and EC) contents in leaves from different sites in tea plant}

The contents of various monomeric catechins were detected in the 'Longjing 43' and 'Baiye 1 hao' cultivars 


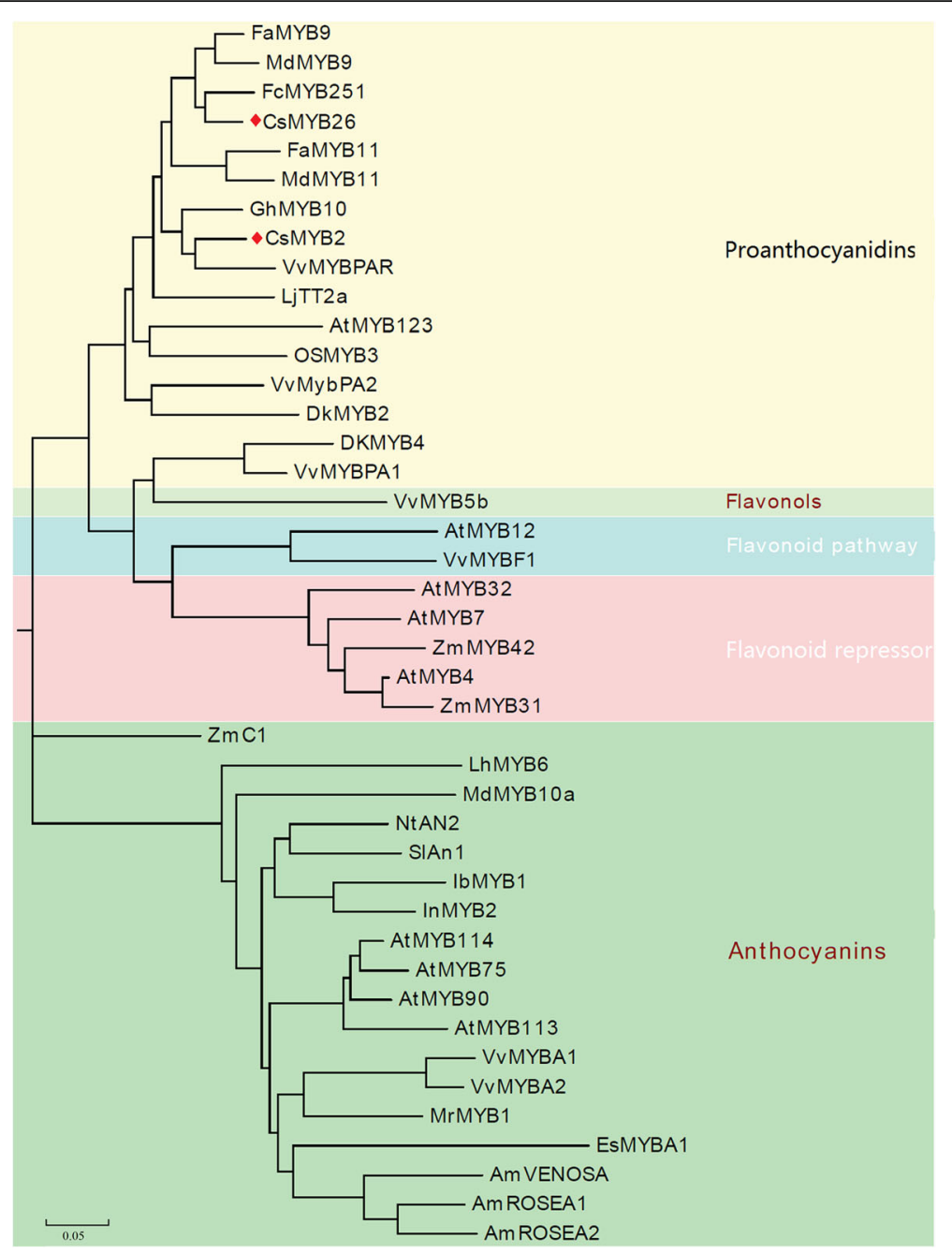

Fig. 3 Phylogenetic relationships among CsMYB2, CsMYB26 and flavonoid-related R2R3-MYBs from other plant species. A phylogenetic tree was built using the neighbor-joining method with MEGA 5 software. The putative functions of all R2R3-MYBs are listed on the right

(Figs. 10 and 11). The content of EC was much higher than that of other components, and the highest content was found in tender leaves followed by old leaves and mature leaves. In addition, the EC content in 'Baiye 1 hao' was higher than that in 'Longjing 43'. The GC content in mature leaves was higher than that in tender leaves. The EGC content decreased in the following order: old leaf $>$ mature leaf $>$ tender leaf, especially in the 'Longjing 43' cultivar. The C monomer was not detected in tender leaves.

\section{Anthocyanidin and PA contents in the leaves from different sites in tea plant}

The content of anthocyanidins and PAs was also detected in the leaves from different sites in the two tea plant cultivars. The anthocyanidin contents were similar in the leaves from different sites of the two tea plant cultivars (Fig. 12a). As shown in Fig. 12b, the content of soluble PAs in the leaves from different sites coincided in the two tea plant cultivars, with the following order: mature leaves $>$ old leaves $>$ tender leaves.

\section{Correlation analysis of CSMYB2 and CsMYB26 levels with those of different structural genes and metabolites}

The correlation coefficients were analysed by correlating CsMYB2 and CsMYB26 expression with that of several structural genes in the flavonoid pathway (Table 1). The results indicated that the correlation coefficients for the expression levels among the CsMYB26, CsLAR, and $C s A N R$ genes were significant. The expression levels of CsMYB26 may thus be positively correlated with those of CsLAR and CsANR. In addition, the expression profiles of CSMYB2 were notably correlated with those of $C s F 3^{\prime} H$ in the two tea plant cultivars. The correlation coefficients of the expression profiles of CsMYB2 and $C s M Y B 26$ with the expression profiles of CsDFR was at 


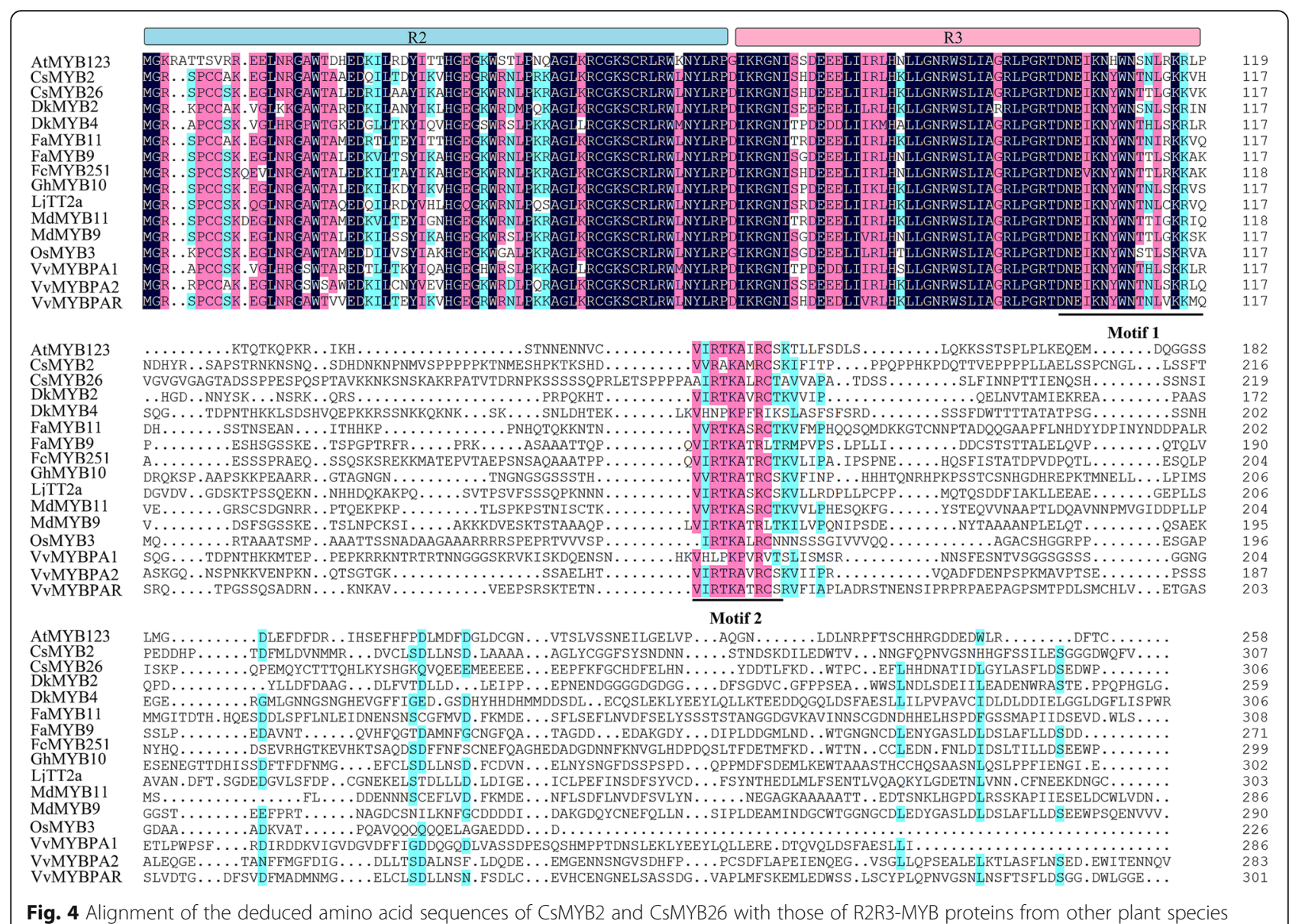

least 0.99 in 'Baiye 1 hao'. Therefore, we speculated that $C s M Y B 2$ and CsMYB26 play a potential role in the regulation of CsDFR expression.

Correlative analyses between the concentrations of flavonoid compounds (catechins, anthocyanins, and PAs) and the expression levels of CsMYB2 and CsMYB26 indicated that the two MYB genes perform differently than those of the flavonoid metabolites. CsMYB2 expression was negatively correlated with GC content and positively correlated with EC content (Table 2). Moreover, CsMYB2

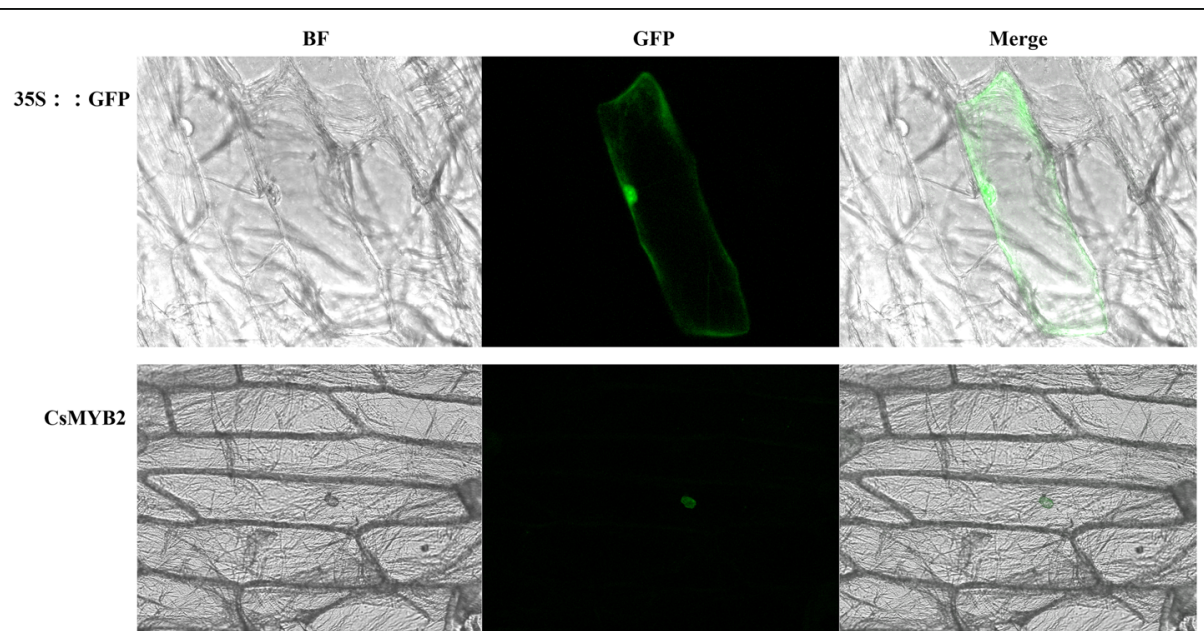

Fig. 5 Subcellular localization of CsMYB2. BF: Bright-field microscopy image. GFP: Green fluorescence image. Merge: Merged bright-field and green fluorescence images 


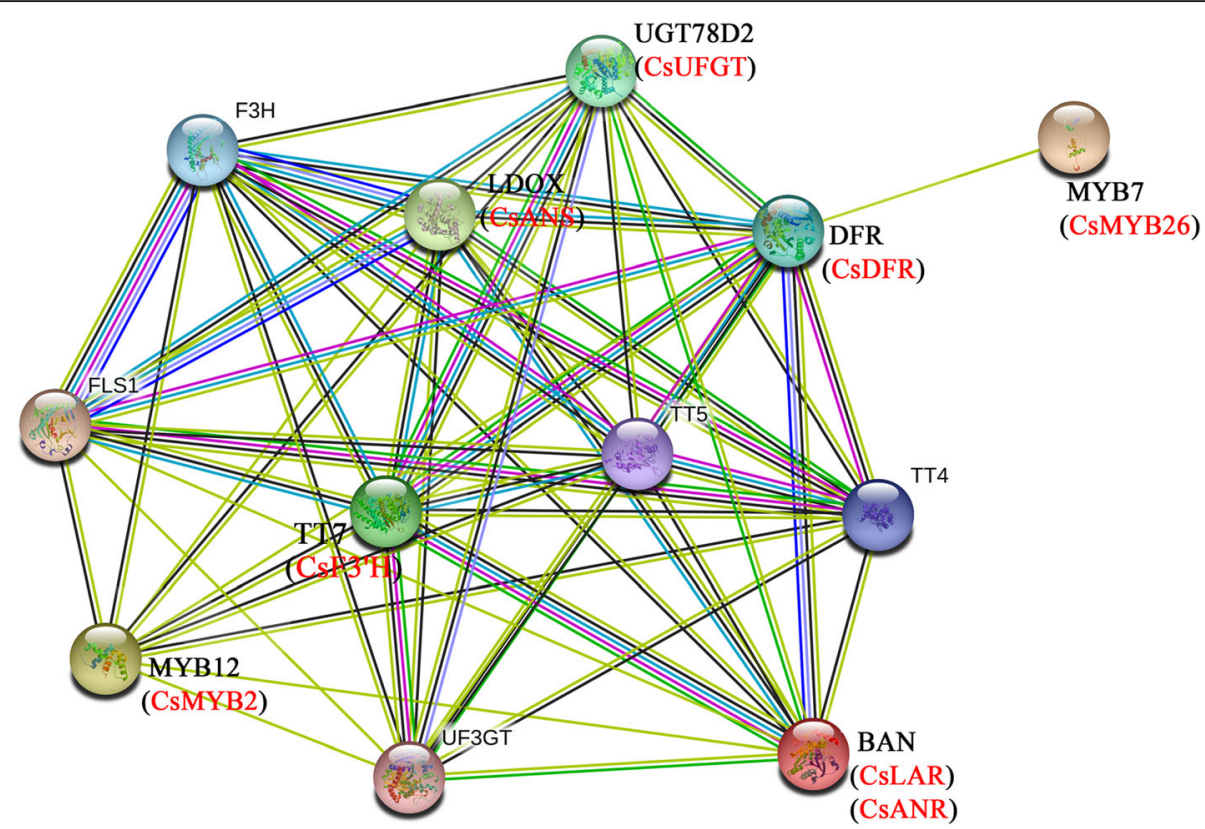

Fig. 6 Interaction network of CsMYB2, CsMYB26 and the structural genes involved in flavonoid biosynthesis

expression was positively correlated with anthocyanin content and negatively correlated with soluble PA content. CsMYB2 positively regulated the content of anthocyanins and negatively regulated the content of PAs in tea plant leaves. CsMYB26 was positively correlated with EGC content and might also regulate anthocyanin content in 'Baiye 1 hao'.

According to the association analysis, CsMYB26 positively regulated CsDRF and CsLAR expression. CsMYB26 also positively regulated $C s A N R$ and $C s F 3$ ' $H$ expression in 'Baiye 1 hao'. Moreover, CsMYB26 expression was negatively correlated with the content of the secondary metabolite EGC. CsMYB2 expression was positively correlated with the expression of CsDFR and CsF3' $H$. CsMYB2 negatively regulated GC content and positively regulated EC content in 'Longjing 43'. In 'Baiye 1 hao', CsMYB2 positively regulated anthocyanin content and negatively regulated PA content.

\section{Discussion}

MYB TFs involved in the flavonoid pathway have been identified in various species. For example, in Arabidopsis, a number of MYB members, including TT2, are widely involved in the regulation of flavonoid pathways [10]. In Fagopyrum, the FtMYB123L gene was used as a homologous gene for $T T 2$ and was found to regulate the flavonoid pathway [17]. To date, the relationship between MYB TFs and the flavonoid pathway in tea plants remains unknown.

In this study, two MYB genes (CsMYB2 and CsMYB26), which are similar to AtMYB123 based on the phylogenetic tree, were identified based on the transcriptome of tea plant [3]. Multiple sequence alignment showed two conserved SANT domains: motif1 and motif2. Motif1 was in contact with the bHLH TF. To date, the function of motif2 is unknown [15]. Phylogenetic analysis suggested that CsMYB2 and CsMYB26 have marked orthologous relationships with VvMYBPAR and FcMYB251, respectively. The CsMYB2 and CsMYB26 TFs were grouped into the PA biosynthesis-related MYB clade. The TT2 gene of $A$. thaliana was found to localize in the nucleus through subcellular localization assays [10]. This finding was consistent with the experimental results of this study, which showed that the CsMYB2 gene also localized in the nucleus.

Our study showed that catechins were mainly detected in old leaves and were almost undetectable in young leaves and mature leaves. EC was mainly found in tender leaves, and its content in old leaves and mature leaves was low [27]. The expression profiles of CsDFR and CSANR were positively correlated with PA content in 'Longjing 43'; however, no such correlation was found in 'Baiye 1 hao'. The biosynthesis and degradation processes involved in the polymerization, esterification, and carbonylation reactions in the flavonoid pathway are very complex and are subject to dynamically changing regulation in the context [28].

According to gene association analysis, a probable regulatory network in CsMYB2 and CsMYB26 is involved in the flavonoid pathway (Fig. 13). The results of the present study suggested that CsMYB2 

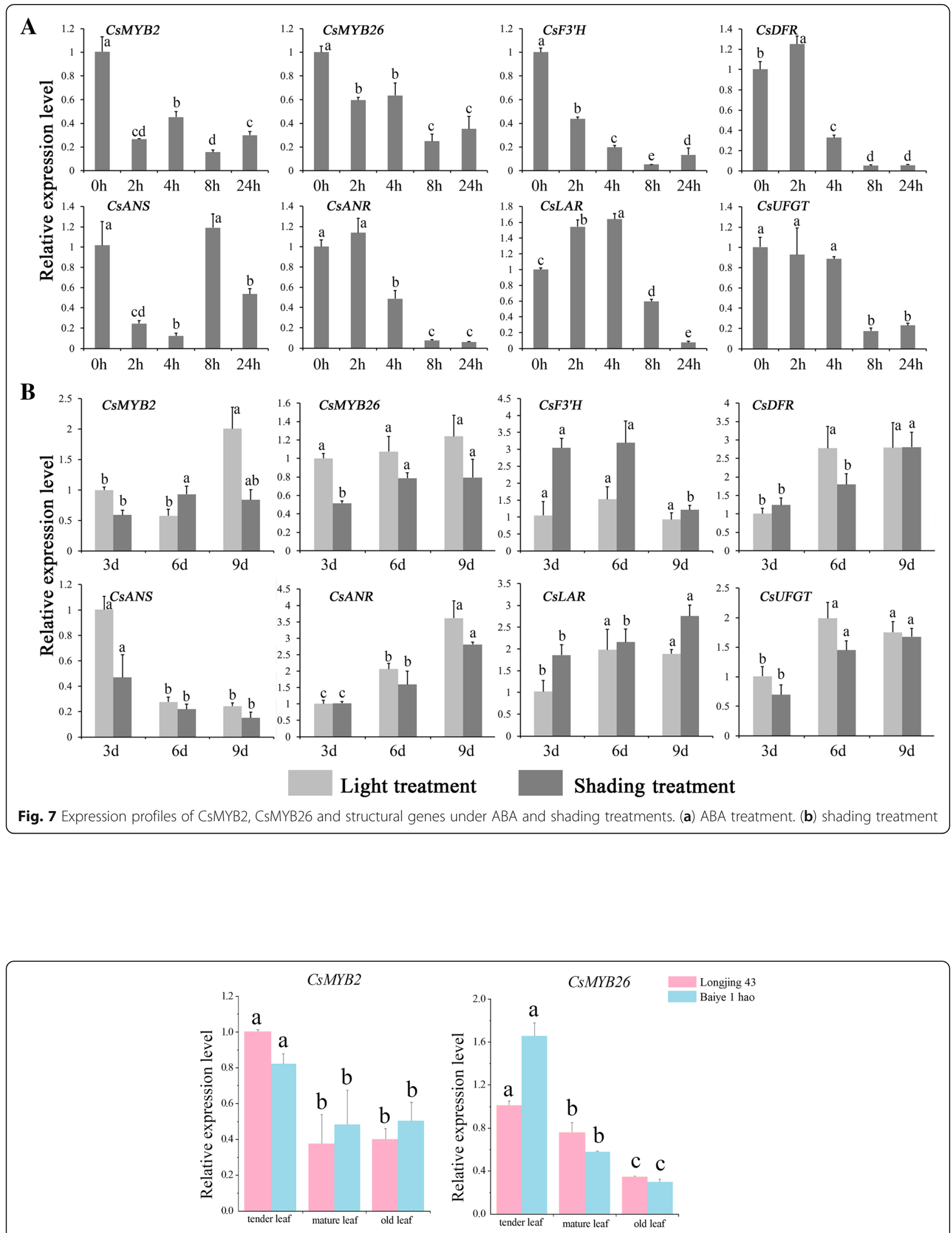

Fig. 8 Relative expression analyses of CSMYB2 and CSMYB26 in the leaves from different sites in tea plant 

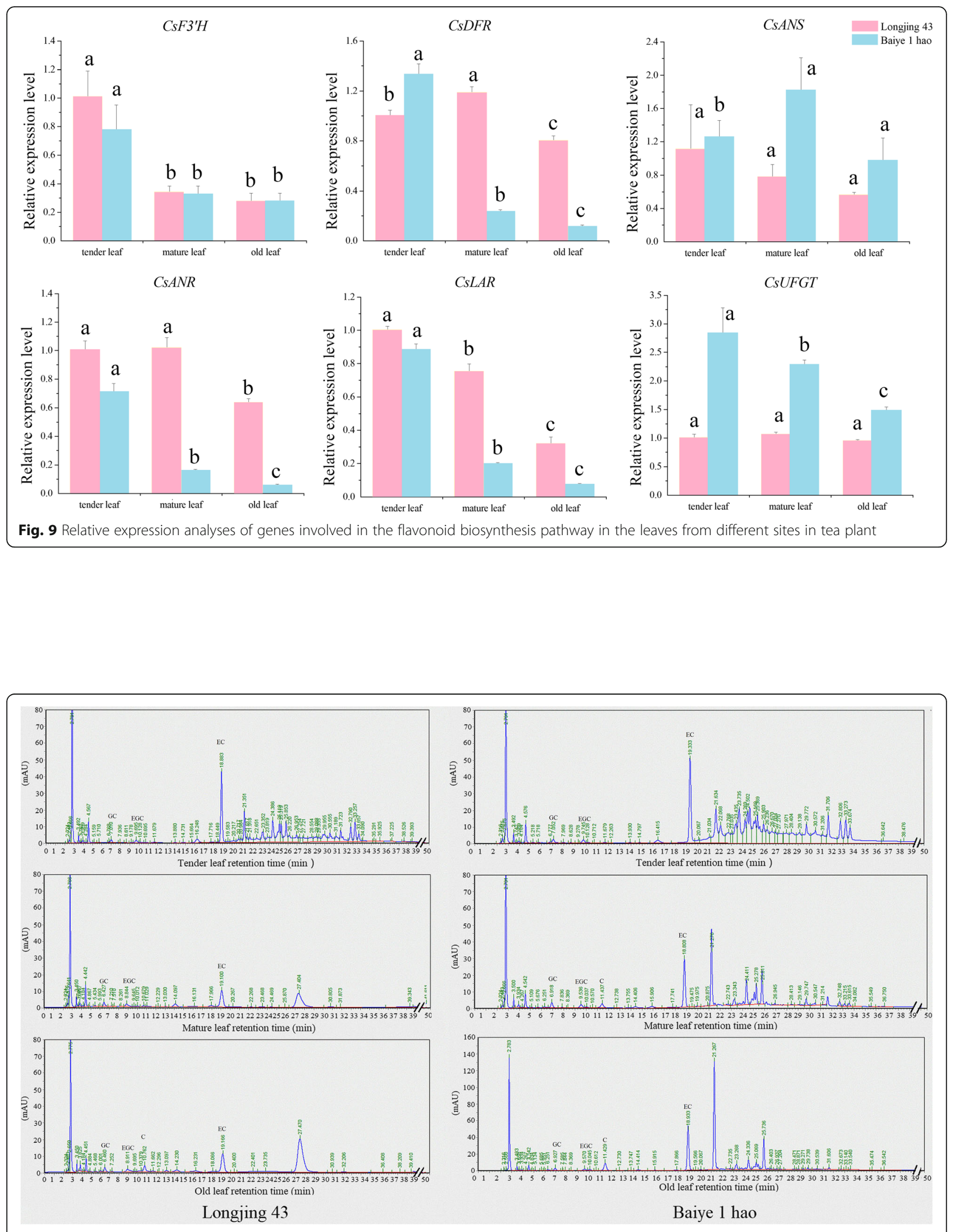

Fig. $10 \mathrm{HPLC}$ chromatogram of catechins in leaves from different sites in the 'Longjing 43' and 'Baiye 1 hao' cultivars 


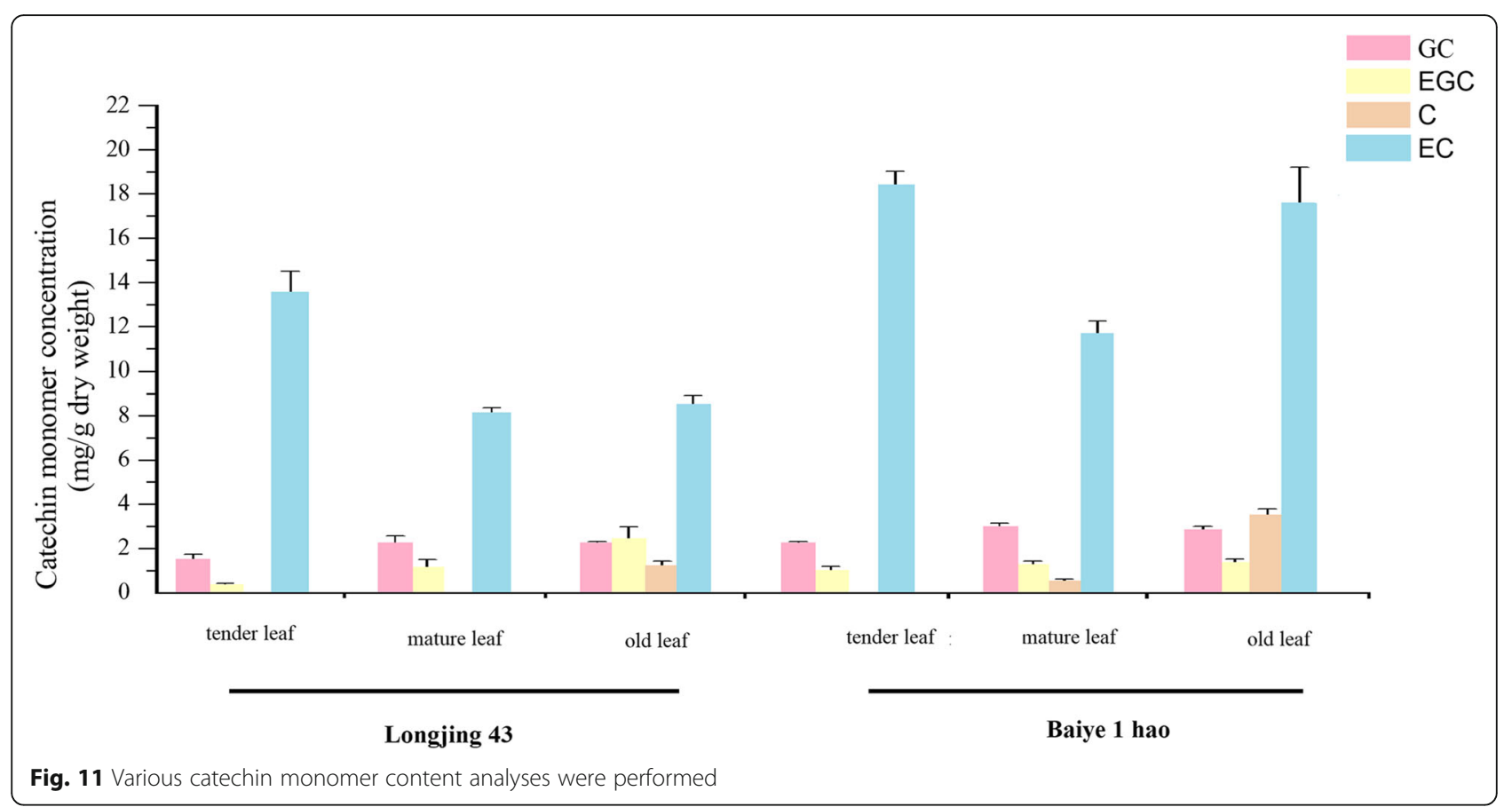

positively regulated $C s F 3^{\prime} H$ in the two tea plant cultivars and that $C s M Y B 26$ expression was positively correlated with CsLAR expression and negatively correlated with EGC content in both tea plant cultivars. This observation was related to the MYB TF levels. Nakatsuka et al. isolated and identified MYB5a and MYB5b, which independently regulate the early steps of flavonoid biosynthesis, resulting in an increase in flavonoid products [29]. As a homologous gene with CsMYB26 and CsMYB2, LjTT2, when overexpressed, increased the expression levels of DFR and ANS and the contents of anthocyanins and PAs in A. thaliana. LjTT2 can directly regulate the late flavonoid pathway [30]. Passeri et al. transferred the $V v M Y B P A 1$ gene of grapevine into $A$. thaliana and found that the increase in PA content was mainly based on the EC-based oligomer composition [18]. Thus, MYB TFs play important roles in the flavonoid pathway.

Promoter analysis indicated that CsMYB2, CsMYB26 and the related structural genes (CsF3'H, CsDFR, CsANS, CsANR, CSLAR, and CsUFGT) contain several MYB recognition elements. The expression of the structural genes might be regulated by $C S M Y B$ genes, and the expression of $C s M Y B 2$ and $C s M Y B 26$ might also be regulated by other $C S M Y B$ genes.

These genes also contain several light- and ABAresponsive elements. The expression of these genes was downregulated under $\mathrm{ABA}$ treatment, indicating that ABA may reduce the expression of structural genes in the flavonoid pathway by regulating the CsMYB2 and CsMYB26 genes, leading to a decrease in flavonoid metabolites. The expression levels of most genes were lower
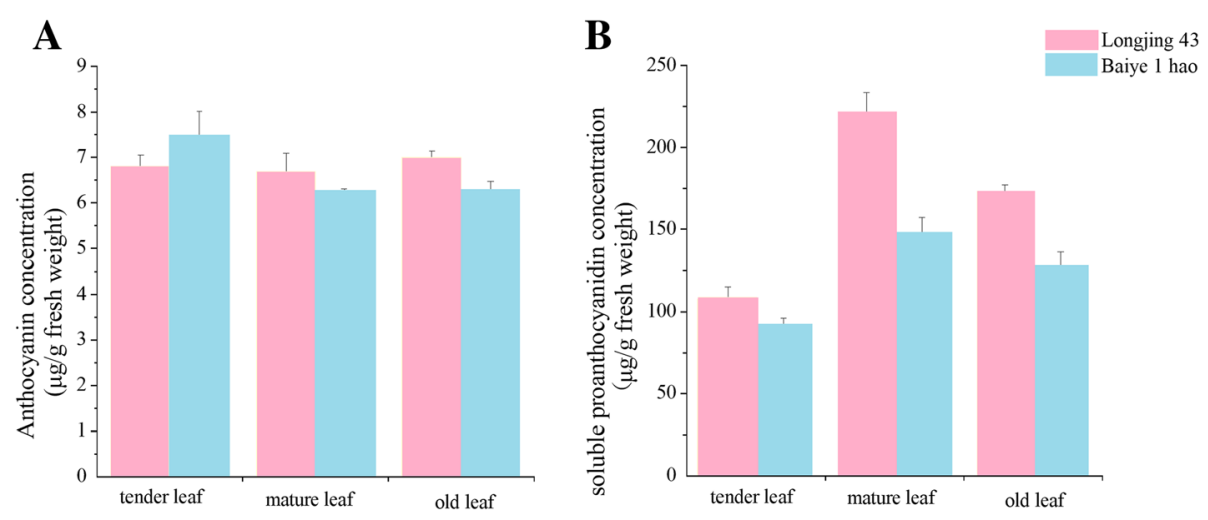

Fig. 12 Content analysis of anthocyanins and soluble proanthocyanidins. (a) Anthocyanin content. (b) Soluble proanthocyanidin content 
Table 1 Correlation of expression levels between regulated genes and related structural genes based on Pearson's correlation analysis

\begin{tabular}{llllllll}
\hline \multicolumn{7}{c}{ Correlation coefficient } \\
\cline { 3 - 7 } & & CsDFR & CsLAR & CSANR & CSANS & CsUFGT & CsF3'H \\
\hline CSMYB2 & Longjing 43 & -0.008 & 0.756 & 0.432 & 0.905 & -0.086 & 0.994 \\
& Baiye 1 hao & 0.99 & 0.982 & 0.98 & -0.242 & 0.78 & 0.99 \\
CSMYB26 & Longjing 43 & 0.642 & $1.000^{\mathrm{b}}$ & 0.914 & 0.965 & 0.58 & 0.828 \\
& Baiye 1 hao & 0.994 & $0.998^{\mathrm{a}}$ & $0.999^{\mathrm{a}}$ & 0.003 & 0.91 & 0.994 \\
\hline
\end{tabular}

${ }^{a}$ Significant correlation at the 0.05 level (two-tailed)

${ }^{\mathrm{b}}$ Significant correlation at the 0.01 level (two-tailed)

under shading treatment than under sunlight treatment, indicating that CsMYB2 and CsMYB26 may regulate and reduce the expression of structural genes in the flavonoid pathway, leading to a decrease in flavonoid metabolites.

\section{Conclusion}

In this study, the content of secondary metabolites in the flavonoid pathway were detected in two tea plant cultivars. The correlation of the expression levels of CsMYB2 and CsMYB26, two R2R3-MYB TFs, and some structural genes involved in the flavonoid biosynthesis pathway were also analysed. The regulatory mechanism governing CsMYB2 and CsMYB26 involvement in the flavonoid biosynthesis pathway was evaluated and predicted. Our findings suggested that $C s F 3$ ' $H$ expression might be controlled by CsMYB2 and that CsLAR expression might be regulated by $C s M Y B 26$. The results also demonstrated that the EGC content was intimately linked to CsMYB26 expression with a negative correlation. Moreover, CsMYB2 and CsMYB26 were confirmed to be involved in the flavonoid biosynthesis pathway. Future studies should explore the possibility of improving flavonoid metabolism via transgenic engineering in tea plant leaves.

\section{Material and methods}

\section{Preparation of plant materials}

The two tea plant cultivars [Camellia sinensis (L.) O. Kuntze cv. 'Longjing 43' and 'Baiye 1 hao'] were deposited in Tea Science Research Institute of Nanjing Agricultural University (Nanjing, China). The 1-year-old vegetatively propagated cuttings of the tea plant cultivars 'Longjing 43' and 'Baiye 1 hao' (Fig. 14) were planted in a phytotron. The relative humidity was programmed at $70 \pm 10 \%$, the temperature was $25{ }^{\circ} \mathrm{C}$, and the light intensity was $300 \mu \mathrm{mol} \mathrm{m} \mathrm{m}^{-2} \mathrm{~s}^{-1}$, with $16 \mathrm{~h}$ of light during the daytime and $8 \mathrm{~h}$ of darkness. Samples were collected with a mixture of corresponding tender leaves, mature leaves and old leaves, immediately frozen in liquid nitrogen, and stored at $-80{ }^{\circ} \mathrm{C}$. The tea plant cultivar 'Longjing 43' was also used for shading and ABA treatment assays. Shade netting ( $60 \% \pm 5 \%$ light transmission) was used for the shading treatment. The tender leaves of tea plant were harvested at 3,6 , and $9 \mathrm{~d}$ after the shading treatment, and tea plants maintained under sunlight conditions were used as controls. In addition, tea plant seedlings were sprayed with $200 \mu \mathrm{M}$ ABA solution, and the tender leaves were harvested at $0,2,4,8$, and $24 \mathrm{~h}$ after treatment. All of the collected samples were immediately frozen in liquid nitrogen and stored at $-80{ }^{\circ} \mathrm{C}$ for further analysis.

\section{Identification of MYB genes in tea plant}

According to the tea database in our laboratory [3], a total of 119 fragments related to R2R3-MYB-type genes were detected, and 27 genes containing two incomplete structural domains were screened out. Furthermore, a phylogenetic tree was constructed based on all R2R3-MYB-type A. thaliana genes and two R2R3-MYB-type TF genes (CsMYB2 and CsMYB26) involved in the flavonoid biosynthesis pathway.

\section{RNA isolation, cDNA synthesis, and gene cloning}

Total RNA was isolated from samples by using a Quick RNA extraction kit (Aidelai, Beijing, China) according to the manufacturer's protocol. Reverse transcription was performed using a PrimeScript RT reagent kit with gDNA Eraser (TaKaRa, Dalian, China), and the samples were then diluted 15-fold in preparation for quantitative real-time PCR (qRT-PCR).

Table 2 Catechin monomers, anthocyanins, and Pas and the expression levels of regulated genes based on Pearson's correlation analysis

\begin{tabular}{llllllll}
\hline & \multicolumn{2}{l}{ Correlation coefficient } & & & \\
\cline { 3 - 7 } & & GC & EGC & C & EC & Anthocyanins & PAs \\
\hline CsMYB2 & Longjing 43 & $-1.000^{\mathrm{a}}$ & -0.77 & -0.471 & $0.999^{\mathrm{a}}$ & -0.101 & -0.912 \\
& Baiye 1 hao & -0.949 & -0.964 & -0.575 & 0.628 & $0.999^{\mathrm{a}}$ & -0.952 \\
\multirow{3}{*}{ CsMYB26 } & Longjing 43 & -0.781 & $-1.000^{\mathrm{b}}$ & -0.93 & 0.739 & -0.722 & -0.429 \\
& Baiye 1 hao & -0.843 & $-1.000^{\mathrm{a}}$ & -0.758 & 0.418 & 0.98 & -0.849 \\
\hline
\end{tabular}

- undetected

${ }^{\text {a }}$ Significant correlation at the 0.05 level (two-tailed)

${ }^{\mathrm{b}}$ Significant correlation at the 0.01 level (two-tailed) 


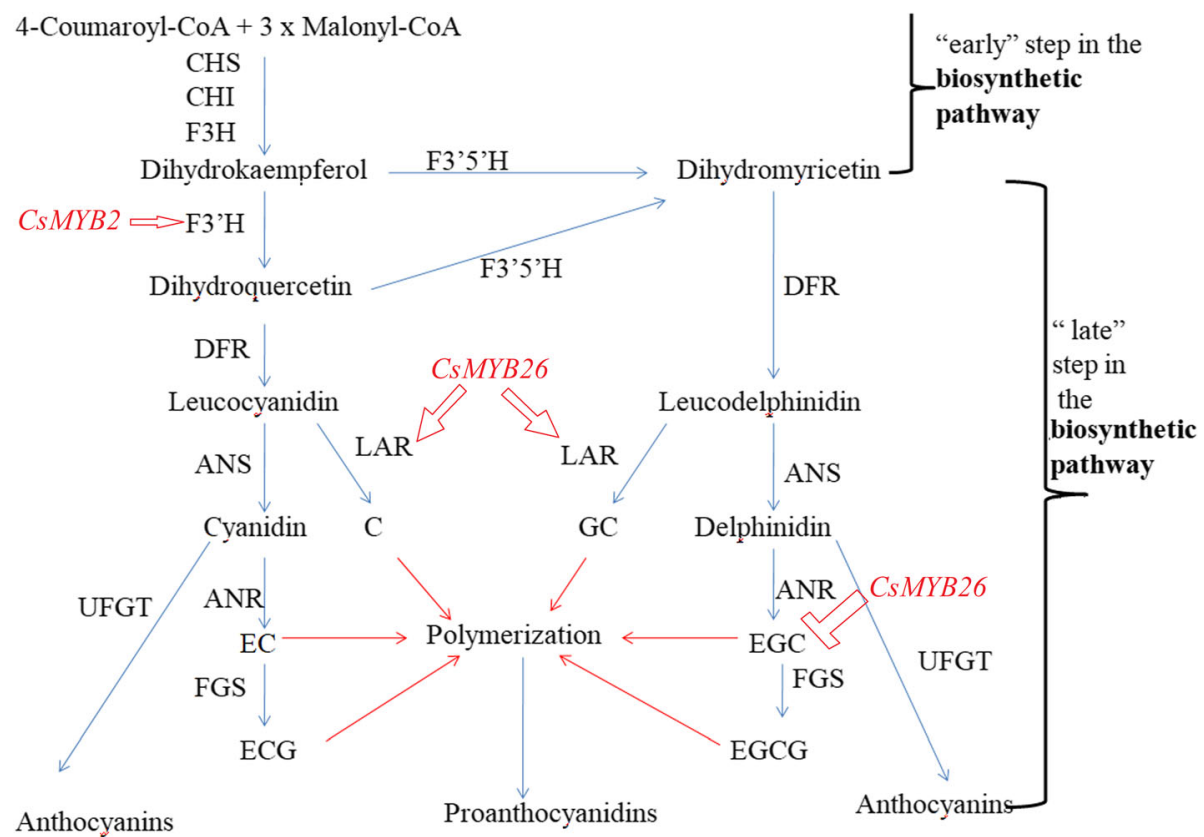

Fig. 13 A possible functional network of the flavonoid biosynthetic pathway and associated regulated genes in tea plant

CsMYB2 and CsMYB26 were amplified from the 'Longjing 43' cultivar, and the primer sequences are listed in Table 3. The PCR program was as follows: $95^{\circ}$ $\mathrm{C}$ for $5 \mathrm{~min}$; followed by 35 cycles at $95^{\circ} \mathrm{C}$ for $30 \mathrm{~s}, 55^{\circ}$ $\mathrm{C}$ for $15 \mathrm{~s}$, and $72{ }^{\circ} \mathrm{C}$ for $60 \mathrm{~s}$; and $72{ }^{\circ} \mathrm{C}$ for $10 \mathrm{~min}$. The PCR products were recovered and inserted into the pMD-19 T plasmid vector (TaKaRa, Dalian, China), and the ligation mixture was transformed into the $E$. coli strain DH5 $\alpha$. Ampicillin resistance was used to identify the clones, which were then sequenced at Tongyong Biotechnology, Inc. (Chuzhou, China).

Multiple sequence alignment, phylogenetic analysis, and conserved motif analysis

The sequences of nucleotides and amino acids were analysed with BLAST on the NCBI website (https://

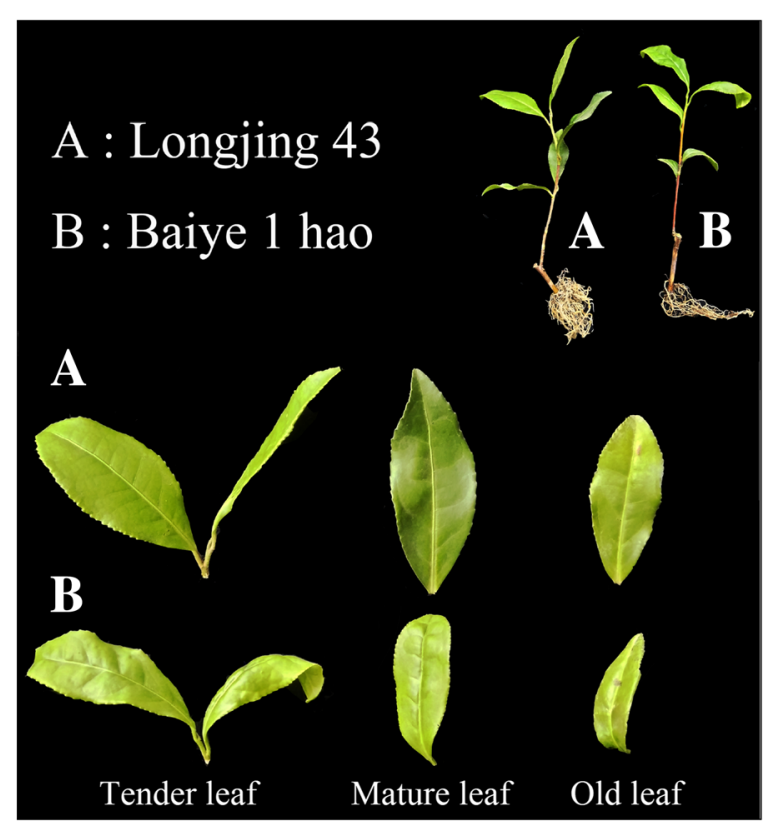

Fig. 14 The two tea plant cultivars. (a) 'Longjing 43' plant. (b) 'Baiye 1 hao' plant 
Table 3 Primers used in the experiments

\begin{tabular}{|c|c|c|c|}
\hline Gene & Direction & Sequence $\left(5^{\prime}-3^{\prime}\right)$ & Function \\
\hline \multirow[t]{2}{*}{ CSMYB2 } & Forward & ATGGGGAGAAGCCCTTGTTGCGCG & Full-length clone \\
\hline & Reverse & CTAGACAAATTGCCAATCCCCTCC & \\
\hline \multirow[t]{2}{*}{ CsMYB26 } & Forward & ATGGGGAGGAGTCCATGCTGC & Full-length clone \\
\hline & Reverse & TCATGGCCAGTCCTCAGAATCAAG & \\
\hline \multirow[t]{2}{*}{ CSMYB2 } & Forward & TTACAGCAATGACAACAA & RT-qPCR \\
\hline & Reverse & CCAGACTCCAGAATAGAA & \\
\hline \multirow[t]{2}{*}{ CsMYB26 } & Forward & СTCCAACTCCATAATATCAA & RT-qPCR \\
\hline & Reverse & GAACAAGGTATCGTCATAA & \\
\hline \multirow[t]{2}{*}{ CSDFR } & Forward & TGCAGAGAGAAGGGTTGCT & RT-qPCR \\
\hline & Reverse & AAGGCAAGGCACCAATACAC & \\
\hline \multirow[t]{2}{*}{ CSANS } & Forward & TCGAGCCCTAGCTACCAAGA & RT-qPCR \\
\hline & Reverse & CAAGTCAGTGTGGGCTTCAA & \\
\hline \multirow[t]{2}{*}{ CSANR } & Forward & GCCTGGTCATGGATGAGAGT & RT-qPCR \\
\hline & Reverse & GGCCATGAGAGTAGGGATGA & \\
\hline \multirow[t]{2}{*}{ CSLAR } & Forward & GGGGCATCCTGTATCAAAGA & RT-qPCR \\
\hline & Reverse & CCGCATACCTTTCAGTCCAT & \\
\hline \multirow[t]{2}{*}{ CSUFGT } & Forward & GCACCATAACCACCCCACC & RT-qPCR \\
\hline & Reverse & TGTCACAAACACACCAACCGAT & \\
\hline \multirow[t]{2}{*}{ CSF3'H } & Forward & CTATTGCAGCTTCTTGATGATCCGA & RT-qPCR \\
\hline & Reverse & GCTCTTTGGTTGCTTTGTTGATTAG & \\
\hline \multirow[t]{2}{*}{ CSMYB2 } & Forward & CACCATCACCATCACGCCATGATGGGGAGAAGCCCTTGTTGCGCG & Subcellular localization \\
\hline & Reverse & CACTAGTACGTCGACCATGGCGACAAATTGCCAATCCCCTCC & \\
\hline \multirow[t]{2}{*}{ GAPDH } & Forward & TTGGCATCGTTGAGGGTCT & Reference gene \\
\hline & Reverse & CAGTGGGAACACGGAAAGC & \\
\hline
\end{tabular}

blast.ncbi.nlm.nih.gov/Blast.cgi). The sequences of AtR2R3-MYB family TFs were downloaded from the PlantTFDB website (http://planttfdb.cbi.pku.edu.cn/) [31]. DNAMAN version 6.0 was utilized to analyse the full alignment. MEGA5 was used to generate the molecular phylogenetic tree with the neighbor-joining method [32].

\section{Analysis of promoter regions and interaction networks of CsMYB2, CsMYB26 and the structural genes involved in the flavonoid pathway}

The functional interaction networks of CsMYB2, CsMYB26 and the structural genes involved in the flavonoid pathway were constructed using STRING software. The sequences 2000 bp upstream of the transcription start site, as promoter regions, were collected from the tea plant genome database (http://pcsb.ahau.edu.cn:8080/CSS/) [33] and submitted to the PlantCARE database (http://bioinformatics. psb.ugent.be/webtools/plantcare/html/) [34] to search for putative cis-acting elements.

\section{Subcellular localization analysis of CsMYB2}

Subcellular localization analysis was mainly performed according to Li et al.'s method with some modification
[35]. Briefly, the CsMYB2 gene was amplified with the stop codons deleted and was inserted into the pA7 vector. The pA7 vector was utilized as a control expressing a 35S::GFP (green fluorescent protein) fusion protein. The constructed plasmids were transferred into onion (Allium cepa) epidermal cells by a helium-driven particle accelerator (PDS-1000, Bio-Rad) and were then incubated for $16 \mathrm{~h}$ at $22{ }^{\circ} \mathrm{C}$ on $1 / 2$ Murashige and Skoog (1/2MS) medium in the dark. The GFP signals were observed using an LSM 780 confocal microscopy imaging system (Zeiss, Germany).

\section{Gene expression level analysis}

A Bio-Rad iQ5 fluorescence quantitative PCR platform was used to perform qRT-PCR with SYBR Premix Ex Taq (TaKaRa, Dalian, China). The experiment was performed in a volume of $20 \mu \mathrm{L}: 10 \mu \mathrm{L}$ SYBR premix, $0.4 \mu \mathrm{L}$ each specific primer, $2 \mu \mathrm{L}$ diluted cDNA, and 7.2 $\mu \mathrm{L} d \mathrm{ddH}_{2} \mathrm{O}$. The cycling conditions for qRT-PCR were as follows: $95{ }^{\circ} \mathrm{C}$ for $3 \mathrm{~min}$, followed by 40 cycles at $95{ }^{\circ} \mathrm{C}$ for $10 \mathrm{~s}$ and $60{ }^{\circ} \mathrm{C}$ for $20 \mathrm{~s}$. All reactions were performed in triplicate. GAPDH was utilized for normalization of the expression levels [27], and the 
$2^{-\Delta \Delta C t}$ method was used to calculate the relative gene expression levels [36]. The primers used in this work are listed in Table 3.

\section{Determination of catechin content from tea plant leaves}

To determine the concentrations of catechin monomers, samples were prepared using the acidified methanol method described by Wu et al. [3]. Briefly, samples of approximately $0.2 \mathrm{~g}$ dry weight were extracted in $5 \mathrm{~mL}$ acetonitrile/water (75:25). The samples were sonicated at room temperature for $10 \mathrm{~min}$ and centrifuged for $10 \mathrm{~min}$ at $5000 \mathrm{rpm}$, and the residues were re-extracted as described above. The collected volume was raised to a total volume of $10 \mathrm{~mL}$. Then, $2 \mathrm{~mL}$ extract was added to $8 \mathrm{~mL}$ of the extract-stabilized solution. Finally, the membrane was filtered through a $0.22 \mu \mathrm{m}$ organic membrane and analysed with HPLC. A $5 \mu \mathrm{L}$ sample was used for reversed-phase HPLC analysis.

\section{Determination of anthocyanin content in tea plant leaves} The anthocyanin extraction protocol was modified based on Pang et al.'s method [37]. Briefly, approximately $0.5 \mathrm{~g}$ mixed tissue samples was ground under liquid nitrogen, extracted with $5 \mathrm{~mL}$ methanol $(0.1 \% \mathrm{HCl})$ for $1 \mathrm{~h}$ with ultrasound, and then centrifuged for $10 \mathrm{~min}$ at $5000 \mathrm{rpm}$. The supernatant was collected, $2 \mathrm{~mL}$ water and $2 \mathrm{~mL}$ chloroform were added to $2 \mathrm{~mL}$ extract to remove chlorophyll, and the sample was then centrifuged for $10 \mathrm{~min}$ at $5000 \mathrm{rpm}$. The supernatant was collected and filtered through a $0.45 \mu \mathrm{m}$ organic membrane, and the absorption of the aqueous phase was measured at $530 \mathrm{~nm}$ and $600 \mathrm{~nm}$. The total anthocyanin content was calculated based on the molar absorbance of cyanidin-3-O-glucoside.

\section{Determination of PA content from tea plant leaves}

As were extracted based on the method of Jiang et al. [38]. Briefly, approximately $0.5 \mathrm{~g}$ mixed tissue samples were ground under liquid nitrogen, extracted with $3 \mathrm{~mL} \mathrm{70 \%}$ acetone $/ 0.5 \%$ acetic acid (extraction solution) by vortexing, and centrifuged for $10 \mathrm{~min}$ at $5000 \mathrm{rpm}$. The residues were re-extracted twice as described above, and the collected volume was raised to a total volume of $10 \mathrm{~mL}$. Then, $2 \mathrm{~mL}$ water and $2 \mathrm{~mL}$ chloroform were added to $2 \mathrm{~mL}$ extract to remove chlorophyll, and the sample was centrifuged for $10 \mathrm{~min}$ at $5000 \mathrm{rpm}$. For the quantification of soluble PAs, $0.5 \mathrm{~mL}$ supernatant was added to a $95 \%$ butanol-HCl mixture. The mixtures were sonicated and boiled for $1 \mathrm{~h}$ and were then cooled to room temperature, followed by centrifugation at $5000 \mathrm{rpm}$ for $10 \mathrm{~min}$. The supernatant was collected and filtered through a $0.45 \mu \mathrm{m}$ organic membrane. The absorbances at $550 \mathrm{~nm}$ and $600 \mathrm{~nm}$ were measured. Cyanidin was used as a standard for the calibration curve. Three technical replicates were performed.

\section{Statistical analyses}

A Pearson correlation coefficient (PCC) analysis was performed to evaluate the correlations between CsMYB2 and CsMYB26 expression levels, the expression levels of 6 structural genes in the flavonoid pathway and the contents of catechin, anthocyanins and soluble PA using SPSS software. $P$-values less than 0.05 were considered statistically significant.

\section{Additional file}

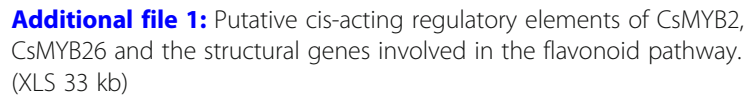

\section{Abbreviations}

ANR: Anthocyanidin reductase; ANS: Anthocyanidin synthase; C: Catechin; $\mathrm{CHI}$ : Chalcone isomerase; CHS: Chalcone synthase; DFR: Dihydroflavonol 4reductase; EC: Epicatechin; ECG: Epicatechin gallate; EGC: Epigallocatechin; EGCG: Epigallocatechin gallate; F3'5'H: Flavanone 3',5'-hydroxylase; F3'H: Flavonoid 3' hydroxylase; F3H: Flavanone 3-hydroxylase; GC: Gallocatechin; GFP: Green fluorescent protein; LAR: Leucoanthocyanidin reductase; NJ: Neighbor-joining; ORF: Open reading frame;"

PA: Proanthocyanidin; PCC: Pearson correlation coefficient; qRTPCR: Quantitative real-time polymerase chain reaction; RP-HPLC: Reversedphase high-performance liquid chromatography; TF: Transcription factor; UFGT: UDP-glucose: flavonoid 3-O-glucosyltransferase; 1/2MS: 1/2 Murashige and Skoog

\section{Acknowledgements}

Not applicable.

\section{Author contributions}

Conceived and designed the experiments: JZ, WLW. Performed the experiments: WLW, YXW, HL, ZWL, XC, JZ. Analyzed the data: WLW, YXW, JZ. Contributed reagents/materials/analysis tools: JZ. Wrote the paper: WLW. Revised the paper: JZ, YXW. All authors read and approved the final manuscript.

\section{Funding}

The work was supported by the National Natural Science Foundation of China (31570691). The funding body does not play any role during the design of the study and collection, analysis, and interpretation of data and in the writing or the decision to submit the manuscript to publication.

\section{Availability of data and materials}

All data generated or analysed during this study are included within the article and its additional files.

\section{Ethics approval and consent to participate} Not applicable.

\section{Consent for publication Not applicable.}

\section{Competing interests}

The authors declare that they have no competing interests.

\section{Publisher's Note}

Springer Nature remains neutral with regard to jurisdictional claims in published maps and institutional affiliations. 
Received: 3 January 2018 Accepted: 26 October 2018

Published online: 20 November 2018

\section{References}

1. Chen Y, Yu M, Xu J, Chen X, Shi J. Differentiation of eight tea (Camellia sinensis) cultivars in China by elemental fingerprint of their leaves. J Sci Food Agr. 2009;89(14):2350-5.

2. Wang $Y$, Gao L, Wang Z, Liu Y, Sun M, Yang D, Wei C, Shan Y, Xia T. Light-induced expression of genes involved in phenylpropanoid biosynthetic pathways in callus of tea (Camellia sinensis (L.) O. Kuntze) Sci Hortic. 2012;133:72-83.

3. Wu ZJ, Li XH, Liu ZW, Xu ZS, Zhuang J. De novo assembly and transcriptome characterization: novel insights into catechins biosynthesis in Camellia sinensis. BMC Plant Bio. 2014;14(1):1471-2229.

4. Jiang XL, Li Z, Li YJ, Liu L, Wu YH, Qian YM, Gao LP, Tan HY, Xia T, Meng F, Wang YS, Li MZ. Analysis of accumulation patterns and preliminary study on the condensation mechanism of proanthocyanidins in the tea plant [Camellia sinensis]. Sci Rep. 2015;5:8742.

5. Feng K, Xu ZS, Que F, Liu JX, Wang F, Xiong AS. An R2R3-MYB transcription factor, OjMYB1, functions in anthocyanin biosynthesis in Oenanthe javanica. Planta. 2017;247(4):1-15

6. Xu ZS, Feng K, Que F, Wang F, Xiong AS. A MYB transcription factor, DCMYB6, is involved in regulating anthocyanin biosynthesis in purple carrot taproots. Sci Rep. 2017;7:45324.

7. Ramsay NA, Beverley JG. MYB-bHLH-WD40 protein complex and the evolution of cellular diversity. Trends Plant Sci. 2005;10(2):63-70.

8. Kranz HD, D M GR, Jin HL, Leyva A, Meissner RC, Petroni K, UA BM, Martin C, Smeekens S, Tonelli C, PA J. Towards functional characterisation of the members of the R2R3-MYB gene family from Arabidopsis thaliana. Plant J. 1998;16(2):263-76.

9. Stracke R, W M, Weisshaar B. The R2R3-MYB gene family in Arabidopsis thaliana. Curr Opin Plant Biol. 2001;4(5):447-56.

10. Nathalie N, C J, Isabelle D, Michel C, Lepiniec L. The Arabidopsis TT2 gene encodes an R2R3 MYB domain protein that acts as a key determinant for Proanthocyanidin accumulation in developing seed. Plant Cell. 2001;13(9):2099-114.

11. Czemmel S, Stracke R, Weisshaar B, Cordon N, Harris NN, Walker AR, Robinson SP, Bogs J. The grapevine R2R3-MYB transcription factor VVMYBF1 regulates flavonol synthesis in developing grape berries. Plant Physiol. 2009;151(3):1513-30

12. Cathie M, Javier PA. MYB transcription factors in plant. Trends Genet. 1997; 13(2):67-73.

13. Sun B, Zhu Z, Cao P, Chen H, Chen C, Zhou X, Mao Y, Lei J, Jiang Y, Meng W, Wang Y, Liu S. Purple foliage coloration in tea (Camellia sinensis L.) arises from activation of the R2R3-MYB transcription factor CsAN1. Sci Rep. 2016;6:32534.

14. Takos AM, Jaffe FW, Jacob SR, Bogs J, Robinson SP, Walker AR. Lightinduced expression of a MYB gene regulates anthocyanin biosynthesis in red apples. Plant Physiol. 2006;142(3):1216-32.

15. Terrier N, Torregrosa L, Ageorges A, Vialet S, Verries C, Cheynier V, Romieu C. Ectopic expression of VvMybPA2 promotes proanthocyanidin biosynthesis in grapevine and suggests additional targets in the pathway. Plant Physiol. 2009;149(2):1028-41.

16. Uematsu C, Katayama H, Makino I, Inagaki A, Arakawa O, Martin C. Peace, a MYB-like transcription factor, regulates petal pigmentation in flowering peach 'Genpei' bearing variegated and fully pigmented flowers. J Exp Bot. 2014;65(4):1081-94.

17. Zhou M, Liang YT, Zhang KX, Li FL, Yang PY, Tang YX, Wu YM, Shao JR. Identification of $\Pi 2$ gene from floral transcriptome in Fagopyrum tataricum. Food Res Int. 2013;54(1):1331-3.

18. Passeri V, DF MS, Carvalho E, Paolocci F. The R2R3MYB VvMYBPA1 from grape reprograms the phenylpropanoid pathway in tobacco flowers. Planta. 2017:246(2):1-15

19. Deluc L, Barrieu F, Marchive C, Lauvergeat V, Decendit A, Richard T, Carde JP, Merillon JM, Hamdi S. Characterization of a grapevine R2R3-MYB transcription factor that regulates the phenylpropanoid pathway. Plant Physiol. 2006;140(2):499-511.

20. Ravaglia D, E RV, Rebecca HK, Andreotti C, Ziosi V, Hellens RP, Costa G, Allan AC. Transcriptional regulation of flavonoid biosynthesis in nectarine (Prunus persica) by a set of R2R3 MYB transcription factors. BMC Plant Biol. 2013;13(1):1471-2229.
21. Feng K, Liu JX, Duan AQ, et al. AgMYB2 transcription factor is involved in the regulation of anthocyanin biosynthesis in purple celery (Apium graveolens L.)[J]. Planta. 2018;248(5):1249-61.

22. Ban $Y$, Honda $C$, Hatsuyama $Y$, Igarashi $M$, Bessho $H$, Moriguchi $T$. Isolation and functional analysis of a MYB transcription factor gene that is a key regulator for the development of red coloration in apple skin. Plant Cell Physiol. 2007;48(7):958-70.

23. Li MZ, Li Y, Guo LL, Gong ND, Pang YZ, Jiang WB, Liu YJ, Jiang XL, Zhao L, Wang YS, Xie DY, Gao LP, Xia T. Functional characterization of tea (Camellia sinensis) MYB4a transcription factor using an integrative approach. Front Plant Sci. 2017:8:943

24. Akagi T, Ikegami A, Tsujimoto T, Kobayashi S, Sato A, Kono A, Yonemori K. DkMyb4 is a Myb transcription factor involved in proanthocyanidin biosynthesis in persimmon fruit. Plant Physiol. 2009;151(4):2028-45.

25. Espley RV, Hellens RP, Putterill J, Stevenson DE, S KA, Allan AC. Red colouration in apple fruit is due to the activity of the MYB transcription factor, MdMYB10. The Plant J. 2007:49(3):414-27.

26. Wang KL, BK GK, Kortstee A, Karunairetnam S, Mcghie TK, Espley RV, Allan AC. An R2R3 MYB transcription factor associated with regulation of the anthocyanin biosynthetic pathway in Rosaceae. BMC Plant Biol. 2010;10(1):50

27. Ashihara H, Deng WW, Mullen W, Crozier A. Distribution and biosynthesis of flavan-3-ols in Camellia sinensis seedlings and expression of genes encoding biosynthetic enzymes. Phytochemistry. 2010;71(5):559-66.

28. Mohammed KA, M J MAD, Bohm JBA, Towers J, HN G. Developmental changes in the composition of Proanthocyanidins from leaves of Sainfoin (Onobrychis viciifola Scop.) as determined by HPLC analysis. J Agr Food Chem. 1993:41(7):1066-70

29. Nakatsuka T, S M, Yamada E, Fujita K, Kakizaki Y, Nishihara M. In Posidonia oceanica cadmium induces changes in DNA methylation and chromatin patternintion factors that regulate early flavonoid biosynthesis. in gentian flower J Exp Bot. 2012;63(2):695-709.

30. Yoshida K, Kume N, Nakaya Y, Yamagami A, Nakano T, Sakuta M. Comparative analysis of the triplicate proathocyanidin regulators in Lotus japonicus. Plant Cell Physiol. 2010;51(6):912-22.

31. Jin J, Tian F, Yang DC, Meng YQ, Kong L, Luo J, Gao G. PlantTFDB 4.0: toward a central hub for transcription factors and regulatory interactions in plants. Nucleic Acids Res. 2017:45(D1):D1040-5.

32. Tamura K, Peterson D, Peterson N, Stecher G, Nei M, Kumar S. MEGA5: molecular evolutionary genetics analysis using maximum likelihood, evolutionary distance, and maximum parsimony methods. Mol Biol Evol. 2011;28(10):2731-9

33. Wei $\mathrm{C}$, Yang $\mathrm{H}$, Wang $\mathrm{S}$, et al. Draft genome sequence of Camellia sinensis var. sinensis provides insights into the evolution of the tea genome and tea quality. Proc Natl Acad Sci U S A. 2018;115(18):201719622.

34. Lescot $M$, Déhais $P$, Thijs $G$, et al. PlantCARE, a database of plant cis-acting regulatory elements and a portal to tools for in silico analysis of promoter sequences. Nucleic Acids Res. 2002;30(1):325-7.

35. Li MZ, L Y, Guo LL, Gong ND, Pang YZ, Jiang WB, Liu YJ, Jiang XL, Zhao L, Wang YS, Xie DY, Gao LP, Xia T. Functional characterization of tea (Camellia sinensis) MYB4a transcription factor using an integrative approach. Front Plant Sci. 2017:8:943.

36. Michaelw P. A new mathematical model for relative quantification in real-time RT-PCR. Nucleic Acids Res. 2001;29(9):e45.

37. Pang $Y$, Peel GJ, Sharma SB, Tang $Y$, Dixon RA. A transcript profiling approach reveals an epicatechin-specific glucosyltransferase expressed in the seed coat of Medicago truncatula. Proc Natl Acad Sci. 2008;105(37):14210-5

38. XI J, Li Y, Li WW, Zhao L, Meng F, Wang YS, Tan HR, Yang H, W C WXC, Gao LP, Xia T. Tissue-specific, development-dependent phenolic compounds accumulation profile and gene expression pattern in tea plant (camellia sinensis). PLoS One. 2013;8(4):e62315. 